\title{
Detection Level of Apple Based on BP Neural Network
}

\author{
Xiaoling $\mathrm{LI}^{1,2}$, Jimin YUAN ${ }^{3}$
}

${ }^{1}$ School of Computer Science and Technology, Chengdu University, Chengdu 610106, P. R. China;

${ }^{2}$ University Key Laboratory of Pattern Recognition and Intelligent information Processing, Chengdu 610106, P. R. China;

${ }^{3}$ The Department Computer Engineering, Chengdu Aeronautic Vocational and Technical College Chengdu 610000, China.

Keywords: characteristic parameter, BP neural network, apple, detect level.

\begin{abstract}
In view of the draw backs of apple grade identification in China, which still relies on photoelectric sorting and manual separation, this paper presents a processing method on the basis of the technology of computer vision and digital image.Utilizing image processing technology, the researcher calculated the length of the long-short-axis, marked the location of it and calculated the 4 parameters, color, mean square,shape,size, as the key characteristics of the BP input of network to build a network and identify the level of apple through analysis of the external characteristics of apple. The optimum structure parameters of the BP neural network which had 9 hidden layer neurons were determined by RP training algorithm. Results showed that average accuracy for fruit classification can reach $92.5 \%$ by using this model, and the executing time of microcomputer for grading of one apple is $9.3 \mathrm{~ms}$. This method has the characteristics of high accuracy and good real-time performance.
\end{abstract}

\section{Introduction}

The usefulness of image detection technology to identify agricultural research has been extensively researched and applied. Rehkugler and He Dong detected defects of apples, utilizing methods of the gray color degree detection of image and color classification[1,2]; ZHAO Maocheng used colored and near-infrared image to analyze the injury area of peaches to classify peaches[3]; LI Ping and Xie zhonghong invented new ways of colors grading on round chilis from the perspective of machine vision, with the correct rate up to 96\%[4,5]; application of computer vision technology to test the quality of apple and surface corruption[6]; and theory of image processing and neural network to determine long fruit, with accuracy rate over $96 \%$ with regard to grading of cucumber levels[7].

At present, there are photoelectric sorting and manual separation with regard to distinguish the grading of apple. Methods of Manual separation were based mainly upon human observation to determine the level. There is lack of objectivity if relying solely on color characteristics and the naked eyes because of the apple sizes and the complex situations of surface. Method of photoelectric separation is based primarily on color characteristics, utilizing surface testing to determine level of apple, which is made up of material delivering equipment, light boxes, electronically controlled lines and pneumatic system to distinguish the colors of Raisin [8-11]. It controls circuits complicatedly and need higher level of users, which restricts its application.

In this paper, image processing and analysis technology are combined with artificial neural networks to identify and grade apple. The key of this way is image manipulation algorithms and characteristic testing, linked to artificial neural networks through extracting valid characteristic parameter[12-14]. The experiment takes Yantai apple as samples.

\section{Image Pre-manipulation}

The photos of apple, $640 \times 480$ pixel, 24-bit true color BMP, as shown in Fig.1 


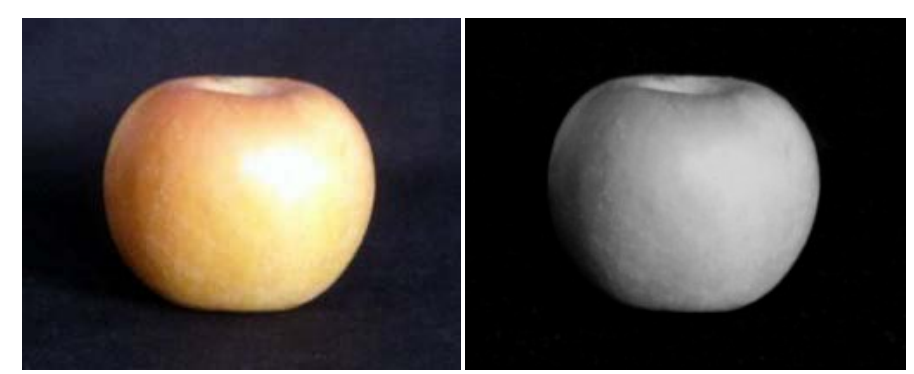

Fig. 1. Apple image (The left: High-grade Apple image; The right: First -grade Apple image)

In the process of image-getting, the quality of image declined because of uneven light and transmission lines etc. So the images need pre-treating for extracting characteristic data. The image Pre-treatment shown in Fig.2.:
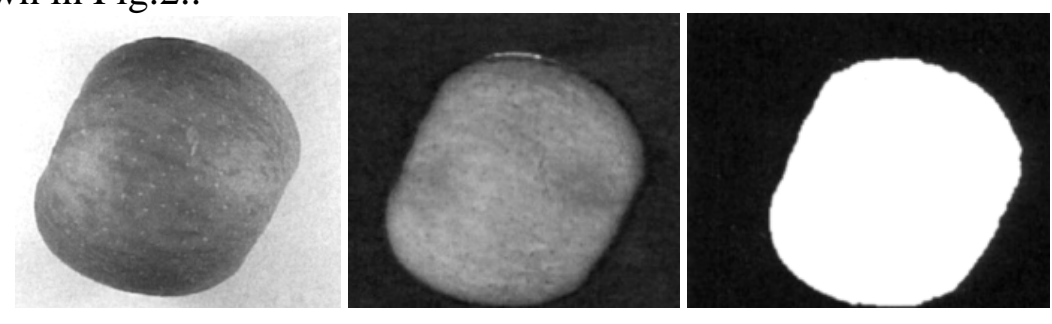

Fig.2. Process of image pre-treatment

In order to identify and grade apple, the characteristics of a single apple need extracting. The following focus on extraction method of color, shape, and other characteristics. Transforming from RGB to HSI can be done as equation1-3.

$I=\frac{R+G+B}{3}$

$H=\cos ^{-1}\left\{\frac{\frac{1}{2}[(R-G)+(R-B)]}{\sqrt{(R-G)^{2}+(R-B)(G-B)}}\right\}$

$S=1-\frac{3 \min (R, G, B)}{R+G+B}$

According to the area feature in the Green theorem , Calculate four levels(each contain 60) of apple samples, and get mean score of 4 characteristics, identify the parameters color, mean square,shape,size as a means of identification features. Because the dimension and value of the above four features have a different order of magnitude, so here be Normalization process shown as equation $4[15]$.

$$
P_{i}=\frac{p-\min (p)}{\max (p)-\min (p)} \quad i=1,2,3,4
$$

\section{Apple-level under BP neural network checkup}

The relation between the form feature and grading is comparatively complex which is hard to distinguish one from another. So we make use of BP network to establish the relationship between form feature and grading which is helpful to distinguish the different grades.

The BP network takes the normalized new vector as input, so the input of BP network is 4 . According to GB / T10651 - 2008, fresh apple level can be divided into excellent , first, second and substandard. So the output nodes are two output layer nodes, where 00 represents excellent , first 
class is 01 , second 10 , substandard grade 11 , representing four different levels of fruit. The input has 4 corresponding feature parameters, while the output is corresponding with the 4 grades of the apple..This paper choose a single hidden layer and number of nodes in the hidden layer neurons can be got from the following empirical formula 5 [16].

$$
n_{1}=\sqrt{n+m}+k
$$

Where $\mathrm{n} 1$ is hidden layer neuron numbers, $\mathrm{n}$ is input layer dimension, $\mathrm{m}$ is output layer dimension, $\mathrm{k}$ is the constant between 1 to 10 .

Known above analysis, the input layer dimension $n=4$, the output layer dimension $m=2$, by the formula $5 \mathrm{n} 1$ should be calculated between 4 to 13 , the specific values can be determined based on the training results. The optimum structure parameters of the BP neural network are determined by RP training algorithm. The 80 samples as training samples are made up of 30 apples in 4 different grades respectively. Network training epochs is set to 10000 , training error is 0.005 , min-grad is1e-10. The training results shown in Table 1.

Table 1 Result of training error under different hidden layer neuron numbers using BP neural network

\begin{tabular}{lllllllllll}
\hline $\begin{array}{l}\text { Hidden-l } \\
\text { ayer }\end{array}$ & 4 & 5 & 6 & 7 & 8 & 9 & 10 & 11 & 12 & 13 \\
\hline RP error & 0.03 & 0.01 & 0.01 & 0.01 & 0.013 & 0.00 & 0.005 & 0.006 & 0.01 & 0.00 \\
& 2 & 4 & 9 & 2 & & 5 & & & 5 & 6 \\
Algorith & 9890 & 1000 & 5403 & 7613 & 1000 & 8118 & 9685 & 2923 & 636 & 545 \\
m & & 0 & & & 0 & & & & 4 & 8 \\
epochs & & & & & & & & & & \\
\hline
\end{tabular}

According to Table 1 , within a predetermined number of epochs, only the hidden layers for 8 and 9 reach the target error. Considering the convergence speed and error, we select 9 as hidden layers .The BP NN grading figure is as Fig.3.

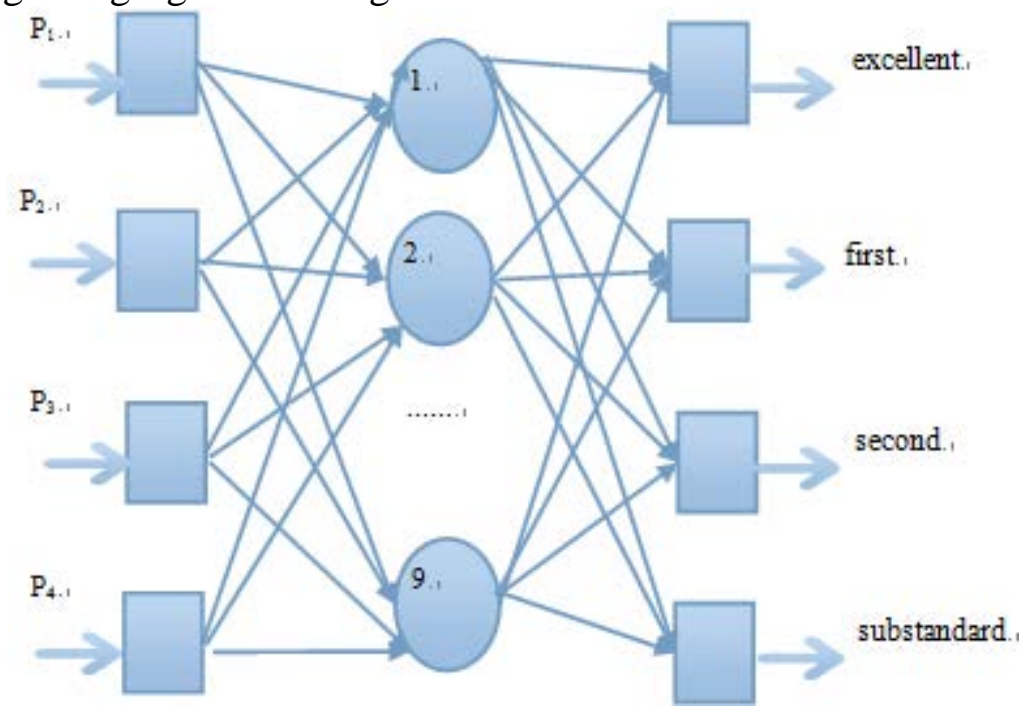

Fig. 3. Grading sketch map of BP neural network

\section{Network training and the results of Recognition}

The experiment is carried out with the help of 4 inputs, 4 outputs and select 120 samples as training samples which are made up of 30 apples in 4 different grades respectively. The training speed is fast, the ratio of identification is very high. The result is shown in table 2. 
Table 1. Table 2 Fruit grades predicted by BP neural network model

\begin{tabular}{lllllll}
\hline $\begin{array}{l}\text { The grade } \\
\text { of training } \\
\text { samples }\end{array}$ & $\begin{array}{l}\text { Artificial } \\
\text { classificati } \\
\text { on }\end{array}$ & $\begin{array}{l}\text { Classification } \\
\text { network } \\
\text { excelle } \\
\text { nt }\end{array}$ & first & $\begin{array}{l}\text { BP } \\
\text { secon } \\
\text { d }\end{array}$ & $\begin{array}{l}\text { neural } \\
\text { substandar }\end{array}$ & $\begin{array}{l}\text { Recognitio } \\
\text { nate of } \\
\text { sample } \\
(\%)\end{array}$ \\
\hline excellent & 30 & 30 & & & & 100 \\
first & 30 & & 27 & 3 & & 90 \\
second & 30 & & 2 & 28 & & 93 \\
substandard & 30 & & 1 & 3 & 26 & 87 \\
\hline
\end{tabular}

From table 2, there are relatively important parameters as color, mean square,shape,size. At the same time, these 4 features are more efficient . Excellent product is with $100 \%$ accuracy , first-class product is with $90 \%$ accuracy , second product is with $93 \%$ accuracy , substandard product is with $87 \%$ accuracy , and all samples are with an average rate of $92.5 \%$ accuracy . The average test time of each fruit $9.3 \mathrm{~ms}$.

\section{Conclusion}

Separating apple is based on image manipulation technology, through the border tracking algorithms. The research put forward to a new method of calculation that test the length of the long-short-axis, marked the location of it and calculated the 4 parameters, color, mean square,shape,size, as the key characteristics of the BP input of network to build a network and identify the level of apple. The result of experiment indicates that the calculating method and judging of the level of apple are precise and accurate, with an average recognition rate of $92.5 \%$.

\section{References}

[1] Rehkugler G E,Throop J A. Apple Sorting with machine Vision.Transaction of the ASAE, 15(4) , pp.1388-1395,1985

[2] He Dongjian, Yang Qing ,Xue Shaoping et al. Computer Vision for Color Sorting of Fresh Fruits. TRANSACTIONS OF THE CHINESE SOCIETY OF AGRICULTURAL ENGINEERING, 14 (2),pp.202-205,1998

[3] ZHAO Maocheng, HOU Wenjun. Method of apple automatic grading based on neural network. Journal of Nanjing Forestry University( Natural Sciences Edition), 33(1),pp.136-138,2009.

[4] LI Ping, ZHU Jun-yan,LIU Yan-de et al. Application and Developing Trend of Computer Version Technology in Detection and Classification of Agricultural Products. Acta Agriculturae Universitis Jiangxiensis. 27(1),pp.796-800,2005

[5] XIE Zhonghong, GUO Xiaoqing, JI Changying. A Met hod of Color Fruit Image Segmentation Based on $\mathrm{t}$ he Color Model and $\mathrm{t}$ he Texture Feature. COMPU TER EN GINEERING \& SCIENCE,132 (11) ,pp:64-66,100,2010.

[6] LIN Wen. Research on apple Appearance Rank Classification Based on Computer Vision and Neural Network. Journal of Anhui Agricultural Science , 38( 23), pp.12703- 12705, $12707,2010$.

[7] ZHANG Rui-yu,LIU Shun-shu. Researches and applications of computer vision technique in fruit and vegetables commercialization after harvesting. Journal of Yuzhou University (Natural Sciences Edition).21(4),pp.497-501,2004.

[8] XIE Feng yun. On Photoelectric Technology of Colour Sorting for Raisin. Journal of Hunan Agricultural University (Natural Sciences).30(6),pp.71-73,2004.

[9] YING Yi bin. Study on background segment and edge detection of fruit image using machine vision[J]. JOURNAL OF ZHEJIANG AGRICULTURAL UNIVERSITY(AGRIC.\& LIFE SCI.).26(4),pp.35-38,2000. 
[10] Chen Li, Luo Xiaoshu, Chen Quanbin. STUDY ON IDENTIFYING SIRAITIA GROSVENORII SPECIES BASED ON IMPROVED BP NEURAL NETWORK. Computer Applications and Software, Vol. 27, No 12 , Dec,2010:240-242.

[11] Tahir A R, Neethirajan S, Jayas D S. Evaluation of the effect of moisture content on cereal grains by digital imageanalysis . Food Research International,40(3),pp.1140-1145,2007.

[12] YAO Li-jian, BIAN Qi, LEI Liang-yu, ZHAO Da-xyu . Classification of fruit based on the BP neural network. Acta Agriculturae Zhejiangensis.24(3), pp.926 -930,2012.

[13] Abdullah MZ, Mohamad-Saleh J, Fathinul-Syahir AS, et al. Discrimination and classification of fresh-cut starfruits (Averrhoa carambola $\quad$ L.) using automated machine vision system. Journal of Food Engineering.76(s ),pp.506-523,2006.

[14] XU Yue-ming, ZHANG Shuang . The Application of Computer Vision on the Classification of Agricultural Products . Journal of Anhui Science and Technology University.26(2) ,pp.8589,2012.

[15] YAO Li-jian, BIAN Qi, LEI Liang-yu, ZHAO Da-xyu.Classification of fruit based on the BP neural network.Acta Agriculturae Zhejiangensis.24(5) pp.926-930,2012. 